\title{
Characterising the Physicality of Everyday Buttons
}

\author{
Jason Alexander \\ Lancaster University, UK \\ j.alexander@lancaster.ac.uk
}

\author{
John Hardy \\ Lancaster University, UK \\ john@highwire-dtc.com
}

\author{
Stephen Wattam \\ Lancaster University, UK \\ s.wattam@lancaster.ac.uk
}

\begin{abstract}
A significant milestone in the development of physicallydynamic surfaces is the ability for buttons to protrude outwards from any location on a touch-screen. As a first step toward developing interaction requirements for this technology we conducted a survey of 1515 electronic push buttons in everyday home environments. We report a characterisation that describes the features of the data set and discusses important button properties that we expect will inform the design of future physically-dynamic devices and surfaces.
\end{abstract}

\section{Author Keywords}

Buttons; Physicality; Input force;

\section{ACM Classification Keywords}

H.5.2. Information Interfaces and Presentation (e.g. HCI):

User Interfaces - Input Devices and Strategies

\section{INTRODUCTION}

Physically-dynamic surfaces-those that can self-modify their geometry-have the potential to revolutionise the touch display industry. One of the key milestones in the development of this technology is the ability to present the user with physical buttons that dynamically appear on the display surface at any position and of any size. To help inform interaction requires and the exploration of accompanying material and engineering challenges, this paper surveys, characterises, and discusses the physical properties of existing everyday push-buttons.

Physical push buttons are pervasive. The properties of these buttons - size, shape, trigger, force, travel distance, audible feedback, etc.- - all contribute to the understanding that certain buttons operate in certain ways, despite their diverse functionality and aesthetic properties. These properties allow distinct groups of buttons to feel similar across devices. For instance, the keys on a computer keyboard are easy to distinguish from those on a washing machine. When absent, this tactile familiarity can lead to an uneasy experience, such as when adopting touch-screens for text input [4].

Interactive systems often emulate the properties of existing objects to build user understanding. To transfer the familiarity and affordances of physical buttons to shape-changing surfaces, it is first important to understand the properties of the Permission to make digital or hard copies of all or part of this work for personal or classroom use is granted without fee provided that copies are not made or distributed for profit or commercial advantage and that copies bear this notice and the full citation on the first page. Copyrights for components of this work owned by others than ACM must be honored. Abstracting with credit is permitted. To copy otherwise, or republish, to post on servers or to redistribute to lists, requires prior specific permission and/or a fee. Request permissions from permissions@acm.org.

ITS '14, November 16 - 19 2014, Dresden, Germany

Copyright 2014 ACM 978-1-4503-2587-5/14/11 \$15.00

http://dx.doi.org/10.1145/2669485.2669519 buttons already in the physical world. To that end, the contributions of this paper are: (1) A detailed survey of over 1500 physical electronic push buttons from 11 different household rooms. Each button is annotated with 20 unique properties, including 5 force profile samples. (2) A characterisation that describes the features of the data set, discusses important button properties, and the relationships between them. The paper concludes with a reflection on the limitations of the collected data and implications for future physically-dynamic surfaces.

\section{RELATED WORK}

\section{Physical Buttons}

Previous physical button investigations have focussed on mechanical push buttons, establishing just-noticeable force differences and user preferences [6], force-displacement transitions [12], keyboard design factors [11], and methodologies for modelling their feel [5]. Our examination adds to work in this area by characterising electronic control buttons that typically have a far shorter input stroke and a greater emphasis on aesthetic design.

\section{Touch-Screen Buttons}

The lack of kinesthetic feedback on touch enabled surfaces has long posed a challenge for interaction designers [4]. The addition of tactile [2] and audio [10] feedback to touchscreen buttons can improve input performance and, along with principles of affective design [15], users' subjective opinion. Tashiro et al. [16] identified buckling and restitution as important tactile characteristics for reproducing the 'click' sensations, with Park et al. [13] using the vibration magnitudes to guide haptically-augmented soft button design.

\section{Buttons on Physically-Dynamic Display Surfaces}

Researchers have produced dynamically available buttons using electromagnetic actuation, pneumatics, and Shape Memory Alloys (SMAs). Early work used linear actuators to displace rods [9] and SMAs [14] to actuate light tubes. Tilt Displays [1] actuate small OLED screens, with the authors postulating that buttons could extrude from the touchscreen. Dynamically adjustable resistance using electromagnetic solenoids can help to prevent typing errors [8].

To create crisp shapes with multiple states and continuous input, Harrison and Hudson [7] used air-filled chambers to make shapes (e.g. buttons) protrude, lie flat, or sink into a display. Rear projection cast an image on pre-defined cutouts, while pressure-sensing was used for continuously variable parameters. Tactus ${ }^{1}$ uses a conceptually similar model, where an overlay on the touch-screen is inflated with liquid.

\footnotetext{
${ }^{1}$ http://www.tactustechnology.com/
} 


\section{CHARACTERISING BUTTONS IN THE PHYSICAL WORLD}

\section{Survey Method}

Everyday environments are full of devices that utilise simple push-button interfaces. We surveyed the physical buttons for electronic controls in 11 typical household rooms, cataloguing a total of 1515 individual buttons. In total we collected 20 measurements for each button, presented here in approximate groupings for clarity:

- Button: Width, height, shape, curvature, texture, material, protrusion above surface, toggability;

- Action: Distance travelled when pressed, force profile required to activate, is the trigger action audible;

- Context: House, room, appliance, function on appliance, number of co-located identical buttons, functions performed by identical buttons, device type (handheld or free standing), degree of sustained use (i.e. a computer keyboard compared to a microwave), similarity of construction material to surroundings.

Button activation force was collected using a FingerTPS ${ }^{2}$ force sensing glove. Each button was pressed five times while recording the forces imparted by the finger. The peak of the activation force and overall profile were extracted. For consistency, a single researcher pressed all buttons, using the index finger on their dominant hand. We achieved an interbutton variance of approximately $100 \mathrm{~g}$ using this method. Button dimensions were measured using a vernier caliper.

\section{Dataset Summary}

The survey profiled 1515 individual buttons from 112 appliances, of which 344 had non-identical characteristics (line 1, Table 1). The most common type of button is rectangular (line 3), flat (line 6), in landscape orientation (line 4), and constructed from plastic (line 7). The vast majority (99\%, line 10) are single state buttons (return to their starting position once triggered) and make an audible noise when pressed (89\%). The mean force required to trigger a button was $326 \mathrm{~g}$ (line 11) although this varied widely across the sample.

\section{Button Characteristics}

We now present a deeper analysis of our sample in order to identify meaningful relationships and interaction between button properties.

Button travel distance is small: The average distance a button travels when pressed is $1.48 \mathrm{~mm}(\sigma: 1.04 \mathrm{~mm})$. Typically, buttons that travel less than $1.00 \mathrm{~mm}$ have a greater peak force applied $(\mu: 494.9 g, \sigma: 534 g)$ than those which move farther $(\mu: 242.7 g, \sigma: 370 g)$. This difference was confirmed statistically significant beyond the $1 \%$ level using a t-test $\left(t_{749.3}=-9.53, p \leq 0.001\right)$. There were no significant sample-wide correlations between button dimensions and distance travelled.

Buttons are seated level with or above the plane: Table 2 details properties of buttons according to their relationship with the surrounding plane. There are a small number $(<1 \%)$

\footnotetext{
${ }^{2}$ http://www.pressureprofile.com/products-fingertps
}

\begin{tabular}{|c|c|c|}
\hline & Characteristic & Value \\
\hline 1 & Buttons surveyed & Total: 1515 , unique: 344 \\
\hline 2 & Appliances surveyed & 112 \\
\hline 3 & Button shapes & $\begin{array}{l}\text { Rectangular: } 71.8 \% \text {, circular: } 15.9 \% \text {, ellipti- } \\
\text { cal: } 9.5 \% \text {, other: } 2.8 \%\end{array}$ \\
\hline 4 & Button orientation & $\begin{array}{l}\text { Landscape: } 62.8 \% \text {, equal dimensions: } 24.1 \% \text {, } \\
\text { portrait: } 13.1 \%\end{array}$ \\
\hline 5 & Pressable surface area & $\mu: 171.7 \mathrm{~mm}^{2}, \sigma: 316.8 \mathrm{~mm}^{2}$ \\
\hline 6 & Curvature & Flat: $74.7 \%$, concave: $15.2 \%$, convex: $10.2 \%$ \\
\hline 7 & Construction material & Plastic: $73.0 \%$, Rubber: $26.4 \%$, Metal: $0.6 \%$ \\
\hline 8 & Appearance & $\begin{array}{l}\text { Differs from surrounds: } 59.7 \% \text {, Identical to } \\
\text { surrounds: } 40.3 \%\end{array}$ \\
\hline 9 & Audible trigger & Yes: $89.1 \%$, no: $10.9 \%$ \\
\hline 10 & Togglability & Full return: $99.3 \%$, toggle: $0.7 \%$ \\
\hline 11 & Input Force & $\mu: 326.4 g, \sigma: 447.2 g$ \\
\hline
\end{tabular}

Table 1. Summary of the push-button dataset

\begin{tabular}{llll}
\hline & Count & Surface Area $\left(\mathrm{mm}^{2}\right)$ & Seating Depth $(\mathrm{mm})$ \\
\hline Below & $12(<1 \%)$ & $\mu: 143, \sigma: 199.6$ & $\mu: 1.65, \sigma: 1.13$ \\
Level & $297(19.6 \%)$ & $\mu: 291, \sigma: 576.6$ & NA \\
Above & $1206(79.5 \%)$ & $\mu: 125, \sigma: 93.5$ & $\mu: 0.89, \sigma: 0.85$ \\
\hline
\end{tabular}

Table 2. Properties of buttons seated above, level, and below the plane

of buttons seated below the plane (line 1), that have, on average, smaller surface areas than those level with (line 2) or above (line 3) the plane. Buttons are on average seated $0.89 \mathrm{~mm}$ below the plane or $1.65 \mathrm{~mm}$ above the plane. Of the 12 buttons below the plane six $(50 \%)$ were to control power to an appliance.

Buttons below the plane are harder to press: Figure 1 shows the peak force distribution of buttons according to their relationship with the surrounding plane. An ANOVA $\left(d f_{2,1512}=3.385, p \leq 0.05\right)$ shows that buttons seated below the plane require greater peak input force $(\mu: 625.4 g, \sigma: 420.9 g)$ than those seated in line $(\mu: 337.0 \mathrm{~g}, \sigma: 601.1 \mathrm{~g})$ or above it $(\mu: 320.57 g, \sigma: 399.7 g)$. This result is in part due to the 'wasted' force a user applies to the surrounding plane when attempting to activate the button.

Button shapes are distributed around a finger's width: Figure 2 provides a frequency-density plot showing that buttons of all shapes tend to be between half a finger and a finger in width [3] (the left peak). A second peak to its right shows a large number of rectangular buttons around widths of $18 \mathrm{~mm}$ - these resemble computer keyboard buttons, which often occur in large numbers. Although the sample contains few buttons with irregular shapes, the distribution indicates that they often have larger and more varied widths than the other types of button. We believe this is due to irregular buttons often forming part of the casing for an appliance.

Landscape orientation is more common than portrait: Viewing buttons in their natural orientation, $62.8 \%$ were landscape, symmetric buttons accounted for $24.1 \%$, and those in portrait orientation just $13.1 \%$. This stems from various design factors including textual button labels and physical selection abilities. In the majority of languages, text is written horizontally (either within the button or below it) requiring more horizontal than vertical space.

On devices with a high-concentration of co-located buttons, landscape orientation can reduce the chance of a press unintentionally triggering multiple buttons as our fingertips have 


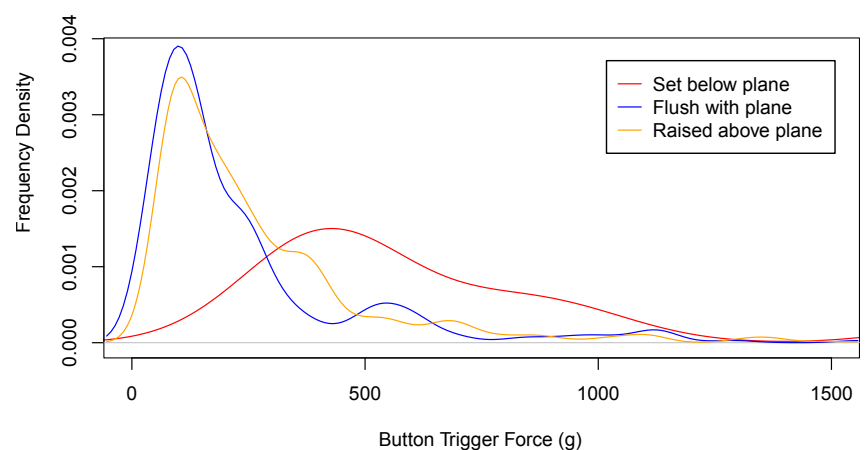

Figure 1. Peak force required to trigger buttons, based on relationship to surrounding plane

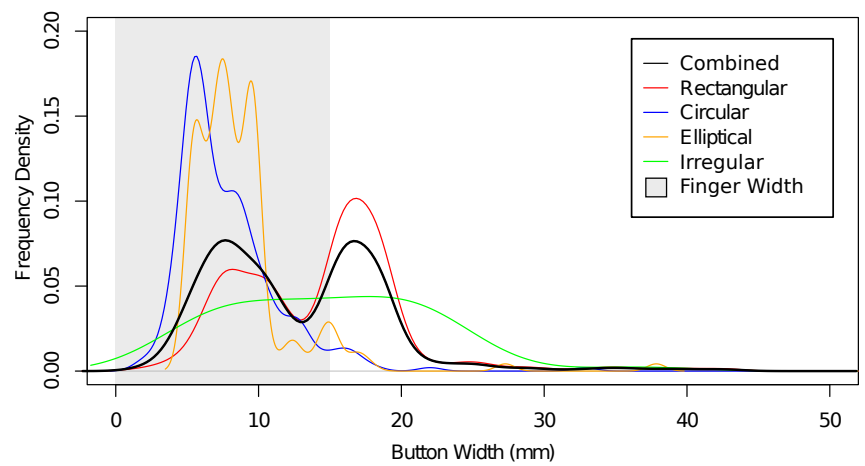

Figure 2. Button width distribution by shape, overlaid with width of a typical adult index finger

a landscape profile. Portrait style buttons requiring fingertip interaction generally result in poor ergonomic design.

Bigger buttons are pressed with less force: Figure 3 shows that most buttons larger than a typical adult index finger $(15.82 \mathrm{~mm} \pm 2.26 \mathrm{~mm})[3]$ are pressed with less force $(\approx$ $100 \mathrm{~g}$ ) than those which are smaller. The smaller buttons are subjected to a much wider range of forces. Although a heteroscedastic t-test indicates that the two groups are significantly different $\left(t_{876.6}=2.564, p \leq 0.05\right)$, we suspect that the higher variance on smaller buttons can be explained by the blunt nature of the finger 'wasting' force on the surroundings. This is supported by the higher force recorded when examining buttons smaller than a finger which are also seated below the surrounding plane.

Longer presses correlate with higher peak force: There is a positive correlation between the interaction time for each press $(\mu: 0.56 s, \sigma: 0.30 s)$ and the peak force reached $\left(r_{1494}=0.73, p<.001\right)$. Figure 4 (left) shows a 'typical' press profile. This is differentiated in Figure 4 (right) to reveal the rate at which the force applied increases.

\section{Buttons with audible feedback require less input force:}

The majority of buttons $(89.1 \%)$ incorporated audible feedback into the trigger mechanism. Buttons with audible feedback require less input force $(\mu: 287.9 g, \sigma: 376.3 g)$ than those without audio feedback $(\mu: 641.8 g, \sigma: 754.2 g)$. A t-test indicates significant differences between the two groups $\left(t_{1513}=9.89, p \leq 0.001\right)$, although examination

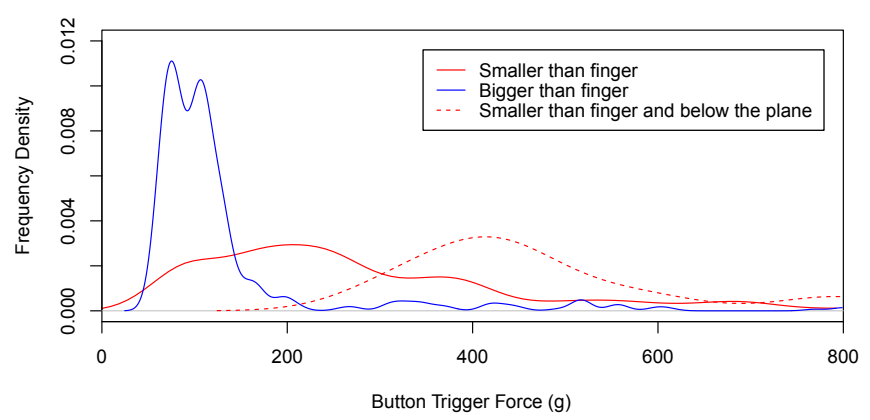

Figure 3. Peak force required to trigger buttons relative to the width of a typical adult index finger
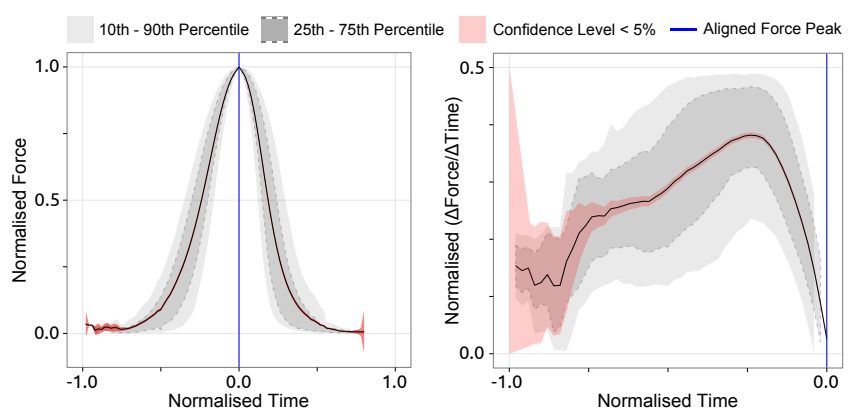

Figure 4. Force and time profile (left) and rate of force increase (right). These are computed by normalising and maxima-aligning all samples

of the distributions indicates additional data would be beneficial. Of the silent buttons, $77.5 \%$ were rubber.

Button input on hand-held devices requires greater force: We classified each appliance as either hand-held or freestanding. Hand-held devices are those which are designed to be held when pressing buttons; Free-standing devices do not require holding under normal operation. During force testing, handheld devices were placed on solid surfaces, to alleviate human variance. People tend to press buttons on hand-held devices with more force, requiring on average $357.5 \mathrm{~g}$ $(\sigma: 363.5 \mathrm{~g})$ to trigger, while freestanding devices received $298.1 \mathrm{~g}(\sigma: 510.2 \mathrm{~g})$ of force. A heteroscedastic t-test shows this difference to be significant $\left(t_{1432.25}=2.625, p \leq 0.01\right)$.

Buttons used in short bursts are smaller: We classified devices based on two degrees of typical user interaction duration: sustained (e.g. games console controllers) and short burst (e.g. a router reset button). Cross-referencing this data with button dimensions revealed two significant groups $\left(t_{1512.8}=-14.5, p \leq 0.001\right)$ where smaller buttons $(\mu: 10.1 \mathrm{~mm}, \sigma: 8.1 \mathrm{~mm})$ are often used in short bursts, and larger buttons $(\mu: 16.5 \mathrm{~mm}, \sigma: 9.2 \mathrm{~mm})$ are used over longer periods of time.

Toggle buttons are rare: We observed only 11 toggle buttons (those that remain depressed once engaged). In the virtual world toggle buttons are commonly used to indicate mode (e.g. when bold is selected in a word processor). In the physical world this mode-indication is often addressed by LED indicators or a physical state change within the appliance. Five of the observed toggle buttons were for power control, the remainder were for function selection. 


\section{DISCUSSION}

\section{Generalisably}

This characterisation has presented a range of features of typical household buttons. The dataset is localised to houses in the United Kingdom with occupants of medium-high socioeconomic standing, meaning a large concentration of modern, electronically controlled devices were present. We observed remarkable consistency across many different types of appliance, acquired for wildly varying tasks (such as washing clothes, drilling holes, or reading ebooks), and see no reason for our findings not to generalise within this group.

The effect a designer has on the properties we measured remains unclear: while some appliances will have undergone much 'button-related' design (such as game controllers and keyboards), others are likely to be influenced by more indirect factors such as cost, manufacturing restrictions, available space, and aesthetics. An inspection of the design and manufacturing process would be required to extract the source of button-design decisions.

\section{Towards Buttons for Physically-Dynamic Displays}

The collected data shows how the physicality of buttons can provide implicit information to the user (e.g. reset buttons are harder to press) and can reflect the needs of the interaction (e.g. lightly tapping keys or pressing small buttons harder ensure activation). Dynamic adjustment of button size and offset relative to the surrounding plane could lead to novel interaction mechanics. E.g., using physical conditions to make actions harder to invoke ('delete friend' or 'power-off').

One of the most important properties for recreating button input in physically-dynamic displays is the ability to reproduce appropriate force profiles based on users' physical world experiences. Short travel distances and the physical sizes of buttons have immediate implications for the resolution and actuation design of materials. Similarly, the observed forceprofiles indicate the required operational range to recreate the experience/familiarity of physical buttons. Physicallydynamic displays can extend this feature to measure the user's input force on-the-fly in order to detect complex input signals, distinguish between intentional and unintentional activation, or detect 'error' conditions where users exert more force than normal in an attempt to reinforce control.

\section{CONCLUSION}

This paper has presented a survey of 1515 physical buttons in household environments. Through our characterisation of these buttons we have extracted a number properties that can guide researchers in the positive transfer of real-world knowledge to physically-dynamic surfaces. We believe the ergonomics ingrained in this data set will continue to be relevant in a generation of physically-dynamic surfaces.

\section{ACKNOWLEDGEMENTS}

This work forms part of GHOST, a project funded by the European Commission's 7th Framework Programme, FET-Open scheme (grant \#309191).

\section{REFERENCES}

1. Alexander, J., Lucero, A., and Subramanian, S. Tilt Displays: Designing Display Surfaces with Multi-axis Tilting and Actuation. MobileHCI '12, ACM (2012).

2. Brewster, S., Chohan, F., and Brown, L. Tactile Feedback for Mobile Interactions. CHI '07, ACM (2007), 159-162.

3. Buryanov, A., and Kotiuk, V. Proportions of Hand Segments. International Journal of Morphology $28(09$ 2010), 755 - 758.

4. Buxton, W., Hill, R., and Rowley, P. Issues and Techniques in Touch-sensitive Tablet Input. SIGGRAPH '85, ACM (1985), 215-224.

5. Colton, M. B., and Hollerbach, J. M. Reality-based Haptic Force Models of Buttons and Switches. In Robotics and Automation '07, IEEE (2007), 497-502.

6. Doerrer, C., and Werthschuetzky, R. Simulating Push-buttons using a Haptic Display: Requirements on Force Resolution and Force-Displacement Curve. EuroHaptics '02 (2002).

7. Harrison, C., and Hudson, S. E. Providing Dynamically Changeable Physical Buttons on a Visual Display. CHI '09, ACM (2009), 299-308.

8. Hoffmann, A., Spelmezan, D., and Borchers, J. TypeRight: A Keyboard with Tactile Error Prevention. CHI '09, ACM (2009), 2265-2268.

9. Iwata, H., Yano, H., Nakaizumi, F., and Kawamura, R. Project FEELEX: Adding Haptic Surface to Graphics. SIGGRAPH '01, ACM (2001), 469-476.

10. Lee, S., and Zhai, S. The Performance of Touch Screen Soft Buttons. CHI '09, ACM (2009), 309-318.

11. Lewis, J., Potosnak, K., and Magyar, R. Keys and keyboards. Handbook of human-computer interaction (1997), 1285-1315.

12. Miller, T., and Zeleznik, R. The design of 3D haptic widgets. In Symposium on Interactive 3D Graphics, ACM (1999), 97-102.

13. Park, G., Choi, S., Hwang, K., Kim, S., Sa, J., and Joung, M. Tactile Effect Design and Evaluation for Virtual Buttons on a Mobile Device Touchscreen. MobileHCI '11, ACM (2011), 11-20.

14. Poupyrev, I., Nashida, T., Maruyama, S., Rekimoto, J., and Yamaji, Y. Lumen: Interactive Visual and Shape Display for Calm Computing. SIGGRAPH '04, ACM (2004).

15. Swindells, C., MacLean, K. E., Booth, K. S., and Meitner, M. J. Exploring Affective Design for Physical Controls. CHI '07, ACM (2007), 933-942.

16. Tashiro, K., Shiokawa, Y., Aono, T., and Maeno, T. A Virtual Button with Tactile Feedback Using Ultrasonic Vibration. In Virtual and Mixed Reality, R. Shumaker, Ed., vol. 5622 of LNCS. 2009, 385-393. 